\title{
Multi-band imaging camera and its sciences for the Japanese near-earth asteroid mission MUSES-C
}

\author{
Tsuko Nakamura ${ }^{1}$, Akiko M. Nakamura $^{2}$, Jun Saito $^{3}$, Sho Sasaki ${ }^{4}$, Ryosuke Nakamura ${ }^{5}$, Hirohide Demura ${ }^{6}$, \\ Hiroaki Akiyama $^{3,4}$, David Tholen ${ }^{7}$, and AMICA team \\ ${ }^{1}$ National Astronomical Observatory, Mitaka, Tokyo 181-8588, Japan \\ ${ }^{2}$ Kobe University, Rokkoudai, Nada, Kobe 657-8501, Japan \\ ${ }^{3}$ Nishimatsu Construction Co., Shimo-tsuruma, Yamato, Kanagawa 242-8520, Japan \\ ${ }^{4}$ Department of Geophysics, University of Tokyo, Hongo, Bunkyo, Tokyo 113-0033, Japan \\ ${ }^{5}$ Earth Observation Research Center, NASDA, Roppongi, Minato, Tokyo 106-0032, Japan \\ ${ }^{6}$ Lunar Mission Laboratory, NASDA, Sengen, Tsukuba, Ibaraki 305-8505, Japan \\ ${ }^{7}$ Institute for Astronomy, University of Hawaii, Honolulu, Hawaii 96822, U.S.A.
}

(Received December 7, 2000; Revised April 23, 2001; Accepted April 23, 2001)

In this paper we present current development status of our Asteroid Multi-band Imaging CAmera (AMICA) for the Japan-US joint asteroid sample return mission MUSES- $\bar{C}$. The launch of the spacecraft is planned around the end of 2002 and the whole mission period till sample retrieval on Earth will be approximately five years. The nominal target is the asteroid 1998SF36, one of the Amor-type asteroids. The AMICA specifications for the mission are shown here along with its ground-based and inflight calibration methods. We also describe the observational scenario at the asteroid, in relation to scientific goals.

\section{Introduction}

Since the discovery of Ceres in 1801 by the Italian astronomer G. Piazzi, ground-based observations have accumulated a vast amount of information on asteroids in various respects. Nevertheless, in-situ asteroid observations from spacecraft are surely expected to provide a unique opportunity for studying some specific asteroids in totally different perspective. In fact, recent observations of the asteroids Gaspra and Ida by Galileo spacecraft (e.g., Belton et al., 1992; Helfenstein et al., 1994; Helfenstein et al., 1996) and of the asteroid Eros by NEAR spacecraft (Veverka et al., 2000) revealed many unexpected new aspects, in terms of such as unusual shape, discovery of a satellite, morphological correlation with surface photometric and colorimetric nature.

Although the discoveries by those US spacecraft that provided significant insight into the origin and evolution of asteroids can never be attained from the ground-based observations, some crucial points like the surface material composition are still left unanswered. This was inevitable because only remote sensing was available for those spacecraft. Hence, there are good reasons to pursue means that can give direct information on the surface material, and the sample return from an asteroid is regarded as one of the best. Considering marvelous achievements of the US Apollo lunar manned missions carried out in 1969-1972 (Kopal, 1974), it is obvious for combination of remote sensing techniques with the sample analysis to give most fruitful results

Copy right(c) The Society of Geomagnetism and Earth, Planetary and Space Sciences (SGEPSS); The Seismological Society of Japan; The Volcanological Society of Japan; The Geodetic Society of Japan; The Japanese Society for Planetary Sciences. in planetary sciences. This motivated us to start the MUSESC mission.

Even if this mission brings about only remote sensing information such as AMICA imaging data, we will still be able to expect qualitatively different results from those for the past US asteroid missions. One reason for the expectation is that, as explained later, the current MUSES-C target asteroid is predicted to have much lower surface gravity due to its estimated small diameter (about one kilometer) than that for the targets of the above US missions (many kilometers in size).

In Section 2, we outline the MUSES-C mission and the target asteroid. Sections 3 and 4 mention the AMICA specifications and its calibrations. And in Section 5, planned scientific goals with the AMICA are introduced in parallel with the observational scenario.

\section{Description of MUSES-C Mission and Target Asteroid}

The MUSES-C is not a pure science mission but basically a technology demonstration mission to a near-Earth asteroid. The project is now under way as a joint program by the Institute of Space and Astronautical Science (ISAS) and the Jet Propulsion Laboratory (JPL) of NASA. The new technological items to be proved in this mission are supposed to be interplanetary ion-engine performance, autonomous spacecraft control at the target asteroid, sample catch from the low-gravity surface, and high-speed Earth reentry. An overall engineering design of the mission was presented in Kawaguchi and Uesugi (1999). The sample return mission from an asteroid is the first attempt in the world that has never been tried before. 
Transfer to 1998 SF36 3/4/4 (in-plane) 1.5year

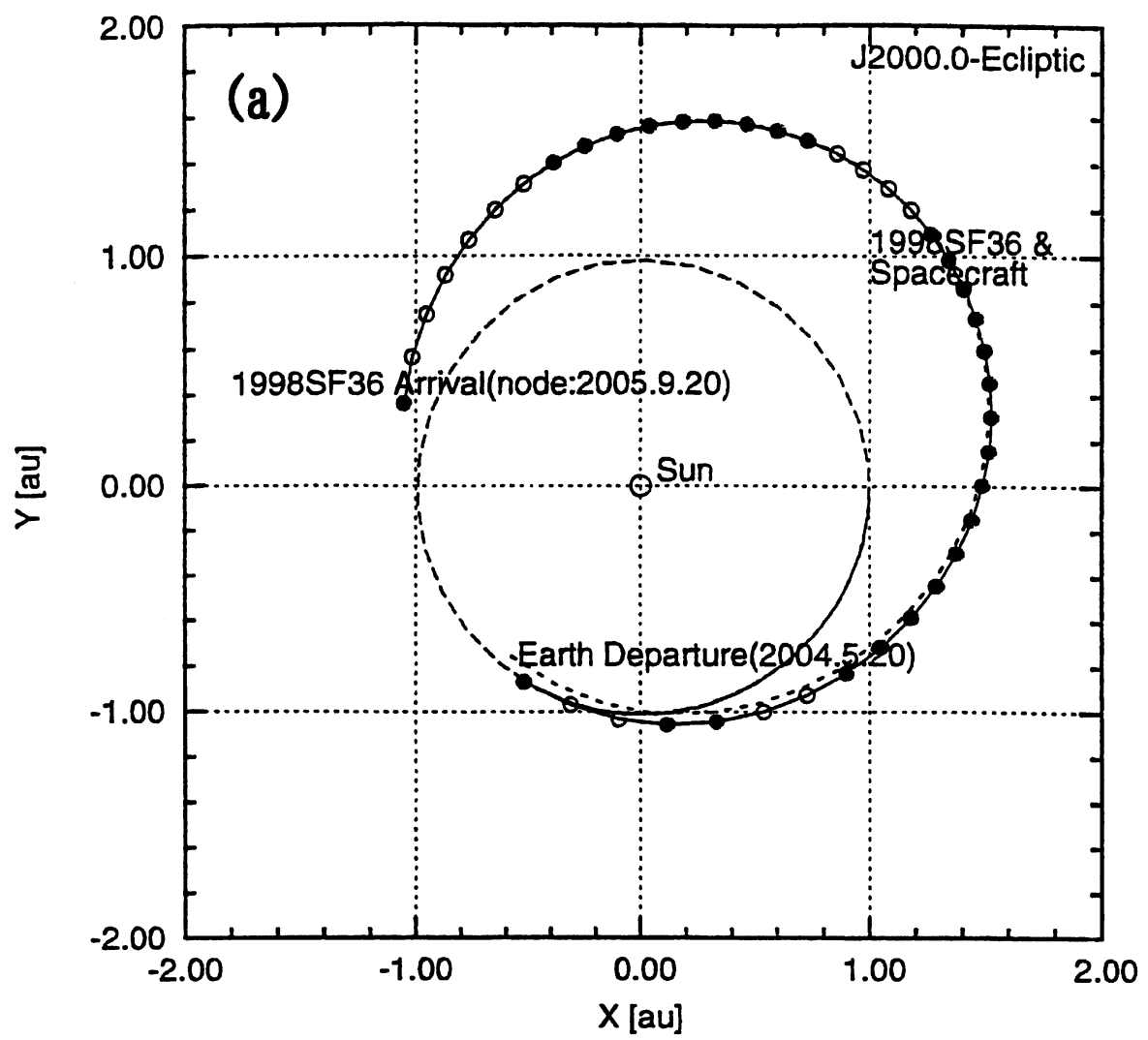

Return from 1998SF36 3/4/4(in-plane) 1.5year

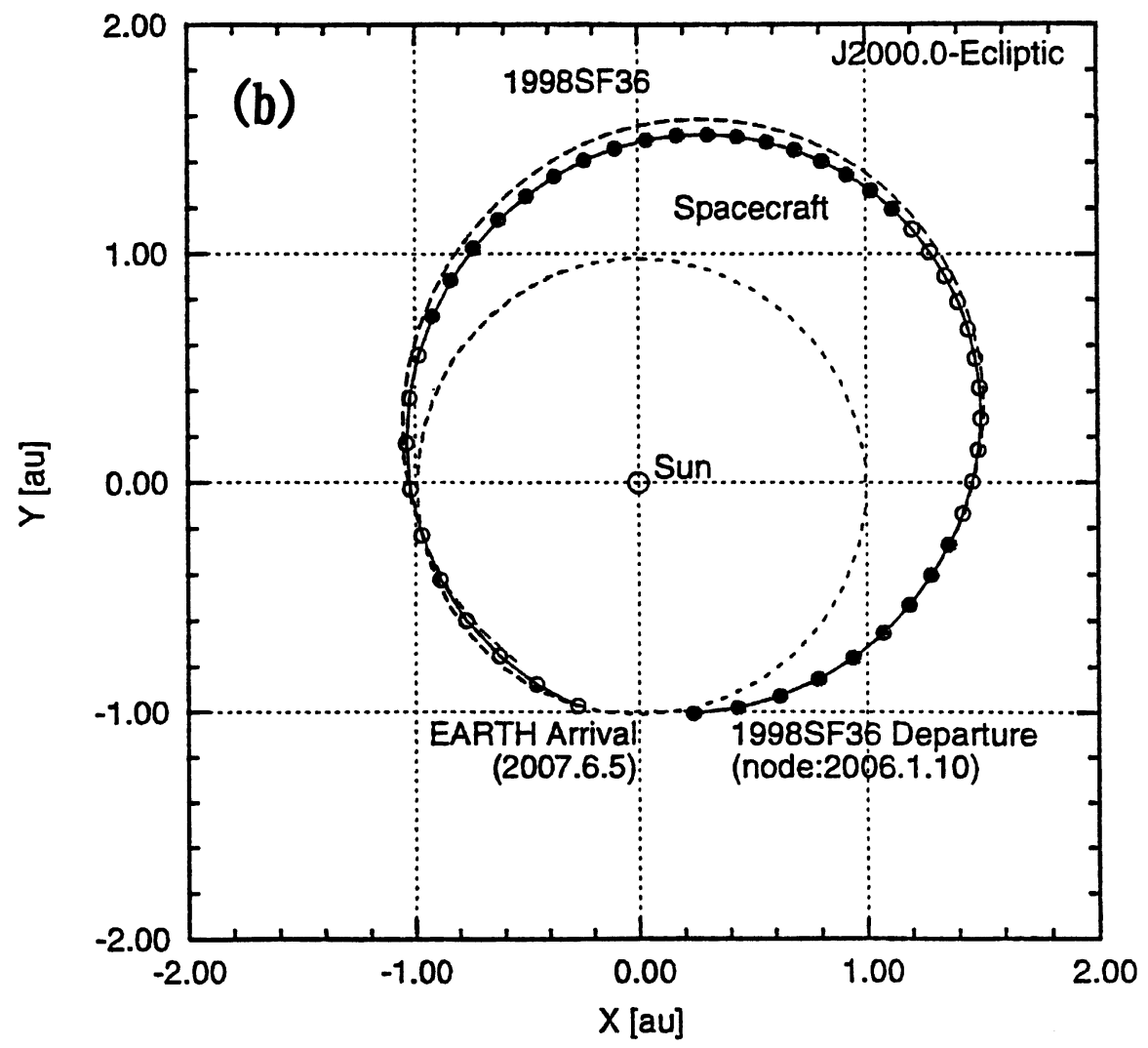

Fig. 1. Transfer (a) and return (b) trajectories for the nominal schedule of the MUSES-C mission to the asteroid 1998SF36. The inner dashed (partially solid) circular curve is the Earth's orbit, and the solid curve with small circles corresponds to the spacecraft's orbit. The filled and open circles stands for "on" and "off" of the ion-engines, respectively. These orbital diagrams are a modified version taken from a document distributed at a certain MUSES-C design conference. 


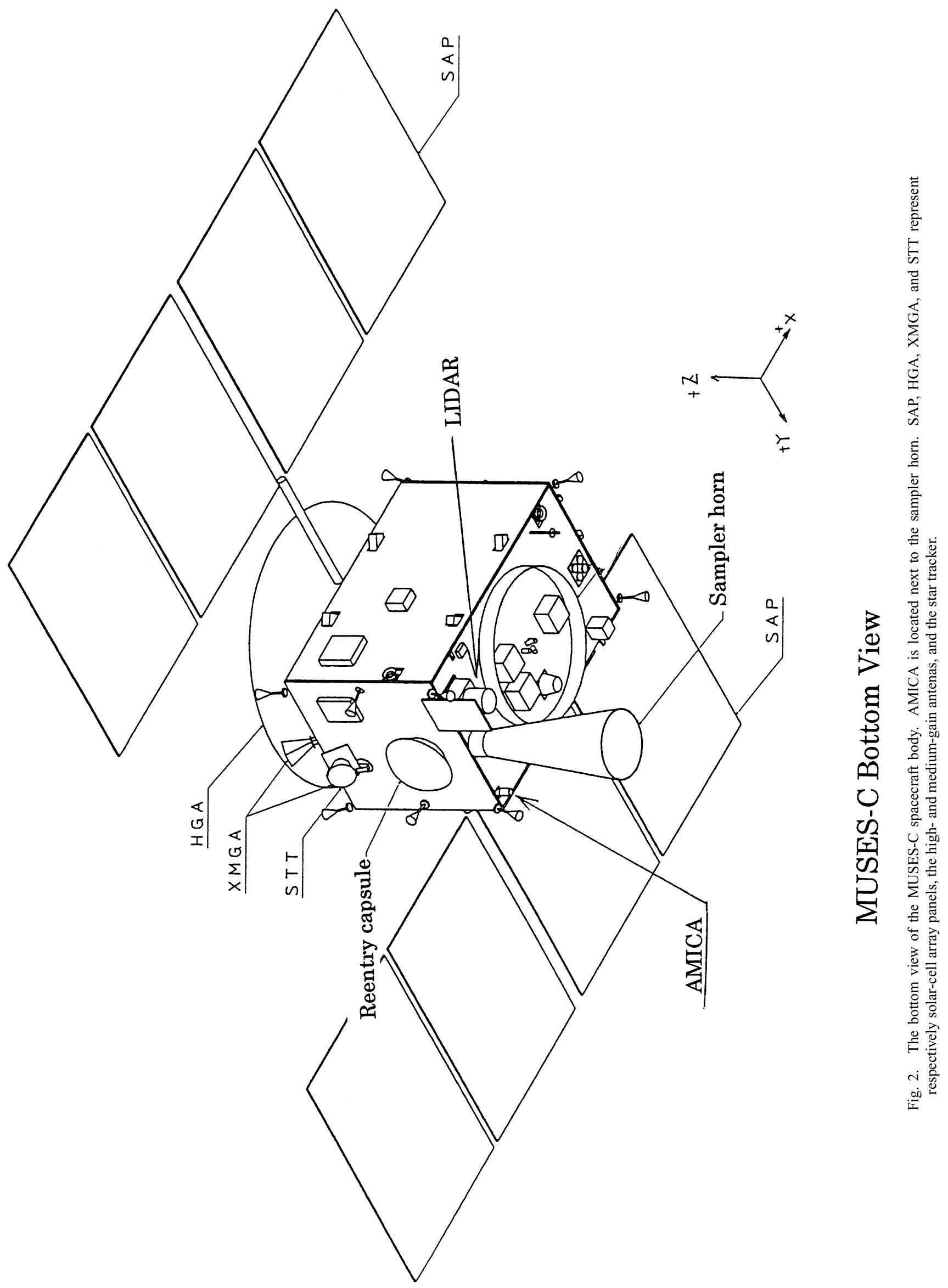




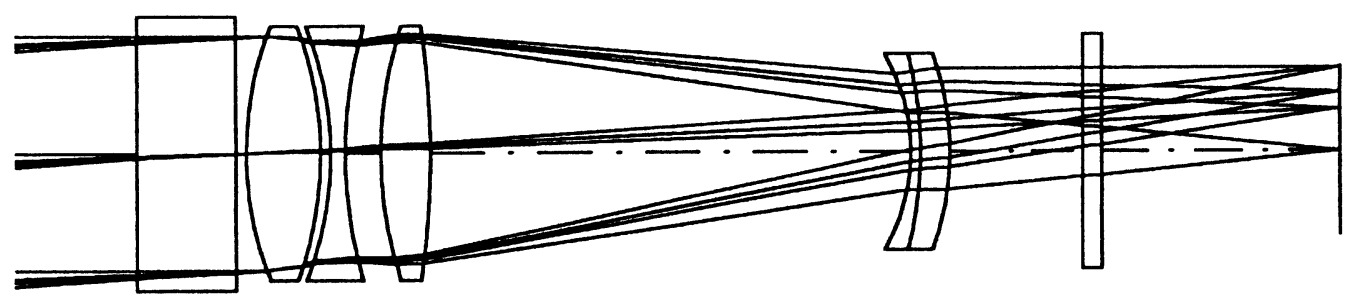

$\mathbf{Q}$

F

CCD

Fig. 3. Optical system of AMICA. A quartz window (Q) is placed in front of the five-lens configuration. F stands for the filter.

Table 1. AMICA specifications.

\begin{tabular}{ll}
\hline Parameter & Specification \\
\hline Weight: Camera/Electronics & $1.74 \mathrm{~kg} / 4.0 \mathrm{~kg}$ \\
Power: Camera/Electronics & $6.8 \mathrm{~W} / 9.5 \mathrm{~W}$ \\
Dimensions & $\mathrm{W} 120 \mathrm{~mm} \times \mathrm{H} 135 \mathrm{~mm} \times \mathrm{L} 180 \mathrm{~mm}$ \\
Lens diameter (effective) & $15 \mathrm{~mm}(\mathrm{~F} / 8.0)$ \\
Focal length & $120 \mathrm{~mm}(\mathrm{fixed})$ \\
Field of view & $5.7 \mathrm{deg} \times 5.7 \mathrm{deg}$ \\
CCD chip & $1024 \times 1000$ pixels (MPP-type $)$ \\
Pixel resolution & $20 \% / \mathrm{pix}(1 \mathrm{~m} / \mathrm{pix}$ at $10 \mathrm{~km}$ height $)$ \\
Quantum efficiency & $45 \% @ 350 \mathrm{~nm}, 20 \% @ 1000 \mathrm{~nm}, 8 \% @ 1050 \mathrm{~nm}$ \\
A/D conversion & $12 \mathrm{bits}$ \\
System spectral response & $360 \mathrm{~nm}-1050 \mathrm{~nm}$ \\
Stellar limiting magnitude & $5-6(\mathrm{~V}-$ band $)$ \\
Exposure time (electronic shutter) & $1 \mu \mathrm{s}, 5.5 \mathrm{~ms}-134$ sec (1.5 times step) \\
Image memory storage & 16 frames \\
Filters & $7-$ bands nearly equivalent to ECAS \\
Polarizers & 4 -position angle glass polarizers \\
\hline
\end{tabular}

Because of this specific characteristic of the mission, it has strongly been felt from the beginning of the project that some scientific key-instruments should be incorporated in the spacecraft, also to justify and maximize the engineering goals. As a result, the AMICA, a near-IR spectrometer, and an X-ray spectrometer were chosen to be onboard through a selection process. One can also use the laser rangefinder (LIDAR) as a scientific instrument, which is a must for navigation near the target asteroid.

The nominal target for the MUSES-C mission is now the asteroid 1998SF36, one of the Amor-type near-Earth asteroids. The semi-major axis, eccentricity and inclination of the asteroid are approximately $1.31 \mathrm{AU}, 0.26$, and $1.7^{\circ}$, respectively, though these values are fairly preliminary (e.g., Minor Planet Electronic Circular 2000t13). Observations at the 2000-2001 opposition will determine the definitive orbit. The only physical information known so far about 1998SF36 is its absolute magnitude $(H), H=18.8$, which translates into a diameter of about $0.6 \mathrm{~km}$ for the average albedo of S-type asteroids. Extensive physical observations will be done near the 2000-2001 opposition to grasp the overall nature of the asteroid.
Figure 1 draws the transfer trajectory (a) and the return trajectory (b) for the nominal schedule of the MUSES-C mission, projected on the ecliptic plane. One can see that the orbit of the asteroid is characterized by a grazing nature to the Earth's orbit. Arrival at and departure from 1998SF36 is planned to be around September 2005 and January 2006, so that the sojourn period at the asteroid is approximately five months. Note that, near the asteroid, the MUSES-C does not orbit around the asteroid but always stays in a heliocentric orbit. This is to save fuel consumption. The captured asteroid sample will be returned to the Earth in the middle of 2007.

Figure 2 shows a bottom view of the MUSES-C spacecraft body, along with the location of the AMICA and some other facilities. AMICA is spacecraft-body-mounted and will be operated to point approximately to the antisolar direction in normal inflight conditions.

\section{AMICA Specifications \\ Hardware}

AMICA system is used for both science and optical navigation purposes. AMICA is basically a simple refractor 


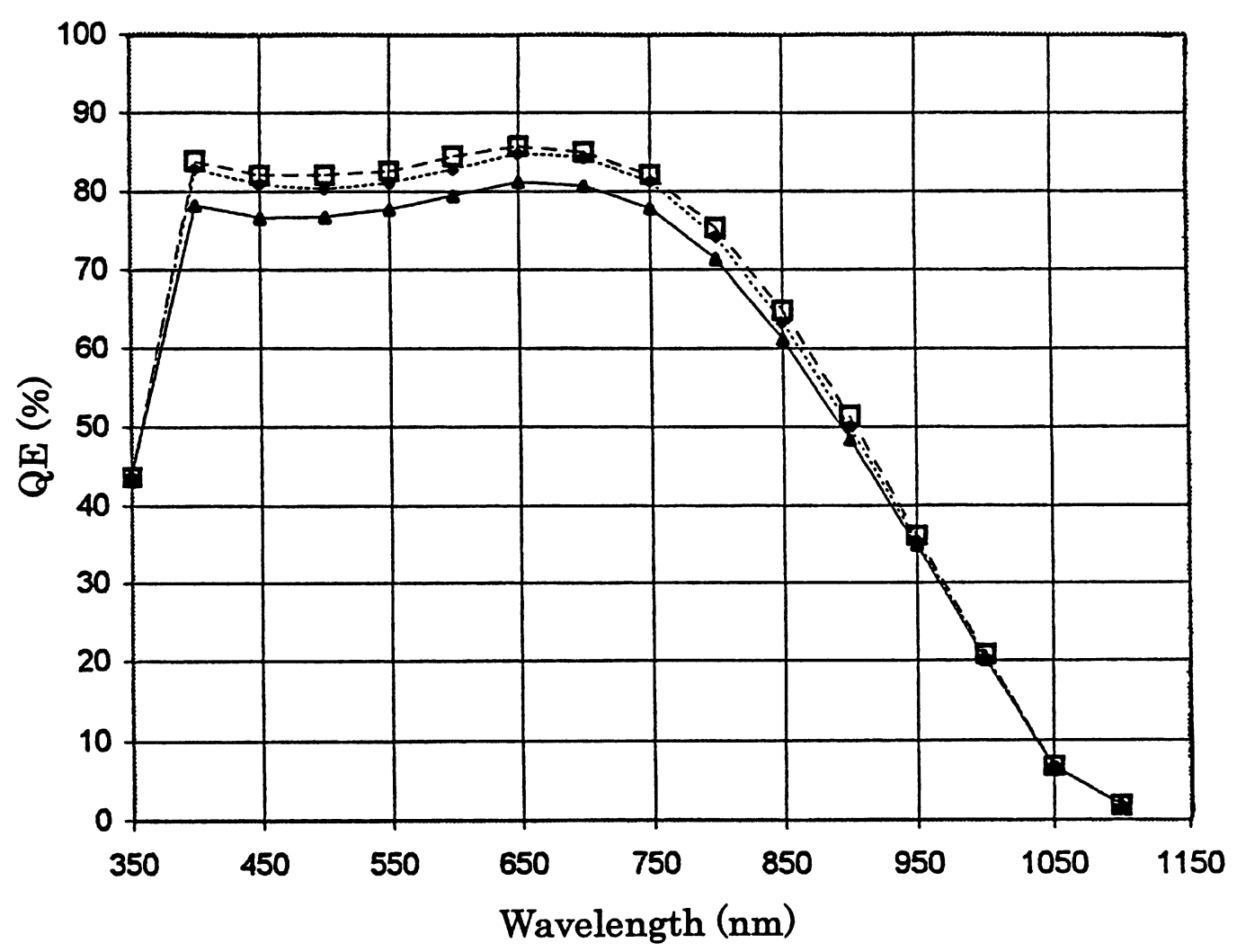

Fig. 4. Measured quantum efficiency (QE) for the AMICA CCD as a function of wavelength. Three curves are measurements for different CCD lots in manufacturing. The CCD used in the AMICA proto-model corresponds to the dotted curve.

telescope consisting of five lenses (Fig. 3), each of which is reasonably cosmic-radiation-resistant and anti-reflectioncoated. Just in front of the first lens, a quartz optical parallel window is placed for radiation protection. The main characteristics of the AMICA is summarized in Table 1.

As seen in the table, the camera field of view is $5.7^{\circ} \times 5.7^{\circ}$ square, which is covered by a CCD of $1024 \times 1000$ pixels, so that the single-pixel footprint corresponds to 20 arcsec, or $1 \mathrm{~m}$ at $10 \mathrm{~km}$ height from the asteroid surface. The adopted CCD chip is of back-illuminated MPP-type (Multi-Pinned Phase), whose dark current is more than two order of magnitudes lower than that for non-MPP chips. This means that the MPP-CCD can be operated nearly equivalently to a nonMPP CCD with a two-stage Peltier cooler. Since the CCD chip of our AMICA is kept below $-10^{\circ} \mathrm{C}$ in ordinary inflight conditions, it corresponds to a non-MPP CCD cooled down to about $-50^{\circ} \mathrm{C}$. Figure 4 represents quantum efficiency $(\mathrm{QE})$ curves as a function of wavelength, for some CCD-chip lots now under development as the proto-model. At wavelengths of 350-400 nm, 10-20\% higher QEs have been attained than that for early test products.

Since our CCD is of a frame-transfer type, the AMICA has no mechanical shutter but the exposure is controlled electronically. We can specify any of 30 different exposure times, ranging from $5.5 \mathrm{~ms}$ through $134 \mathrm{~s}$ including $0 \mathrm{~s}$ exposure (precisely, less than $1 \mu \mathrm{s}$ ). Such a wide coverage of exposure time was needed to meet both the opposing requirements, a short exposure for fully illuminated surface of the asteroid and a long one for faint ECAS standard stars.
The 0 s-exposure is also necessary to correct readout smear which is inevitable for shutterless and frame-transfer type CCDs.

AMICA is equipped with an eight-position filter wheel. As shown in Fig. 5, seven filters are nearly equivalent to the ECAS bands (Tedesco et al., 1982). This choice is because the ECAS system is a multi-band photometric system specially designed for asteroids, with which many asteroids have so far been measured for taxonomic classification (Tholen, 1984; Zellner et al., 1985). Inflight calibration of the AMICA filters using the ECAS standard stars (Tedesco et al., 1982) will open up a way of directly comparing the surface colors of the target asteroid with the abundant database of the ECAS asteroids (Zellner et al., 1985). In the eighth position is a closeup lens with v-band filter, which is intended to be used for imaging near the touchdown phase for sampling. Each filter aperture is sectorshaped and placed closely side by side on the edges. This prevents us from becoming blind when the filter wheel stops by accident at an unexpected position.

AMICA also has four position-angle glass polarizers. They are located on one edge of the CCD chip (covering $200 \times 200$ pixels each), immediately in front of it. This allows us to measure polarization of the asteroid in the vand w-bands (transmission of the glass polarizers tends to zero for bands shorter than $500 \mathrm{~nm}$, and contrast becomes severely low toward bands longer than $900 \mathrm{~nm}$ ). Because of difficulty in delicate attitude control of spacecraft, we assume that the simple polarimeter only works as a photometer 


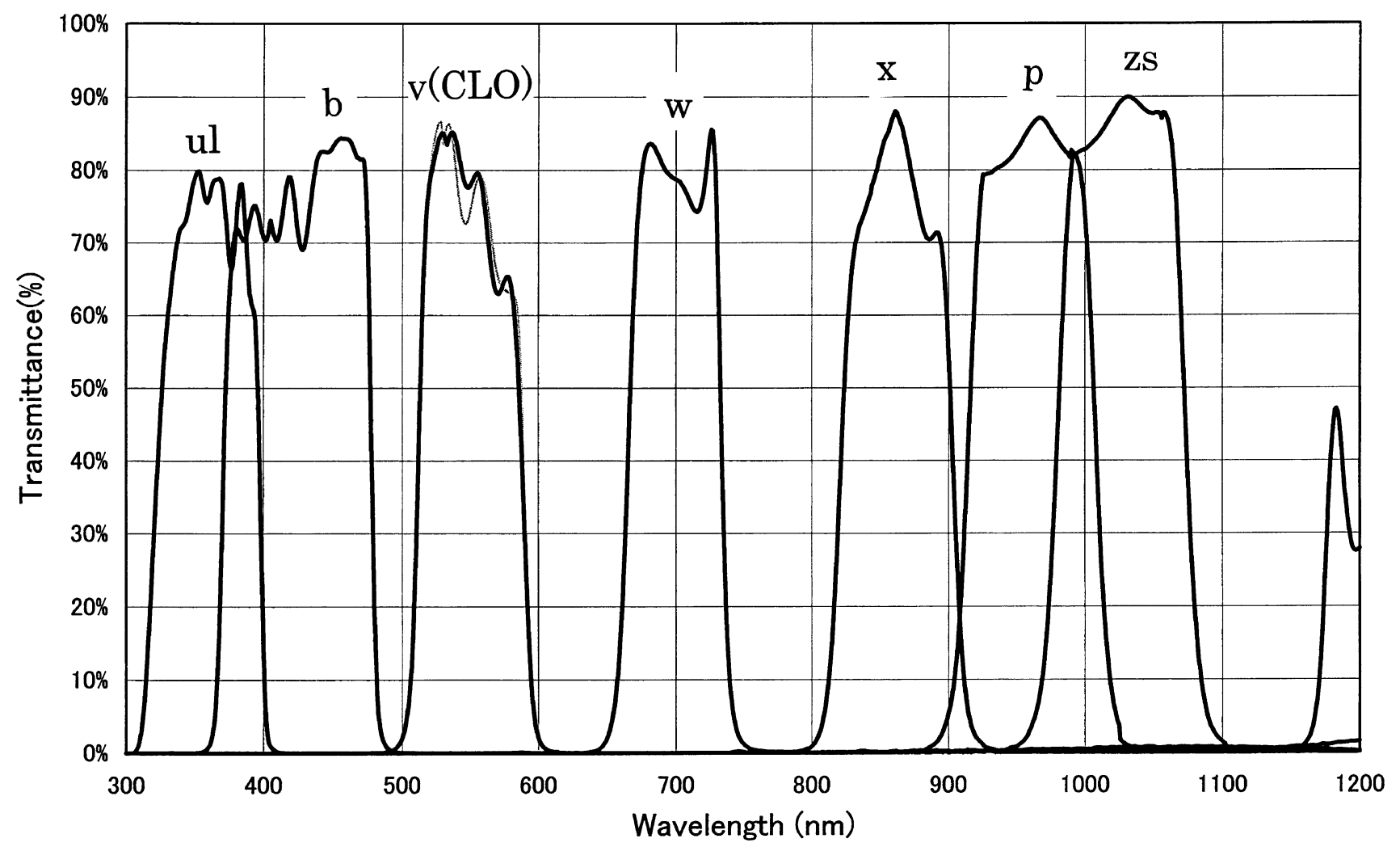

Fig. 5. The filter system of AMICA. Seven filters are nearly equivalent to the ECAS bands, namely ul-, b-, v-, w, x-, p-, and zs-filters. At wavelengths longer than $1150 \mathrm{~nm}$, there is a 2nd-order band-leakage caused by v-filter. However, this has no effects in asteroid photometry, because the CCD has no sensitivity in this wavelength region (see Fig. 4). Around 500-600 nm, the thick and thin solid curves correspond to the v-filter and that for the closeup lens (CLO), respectively.

rather than an imager.

In Fig. 6 is given a photo of the assembled AMICA protomodel. The large cylinder seen in the middle is the filter wheel casing, and the black box in the back is the electronics container for the camera control and data handling. The black tube in the front includes the telescopic part. Two protrusions located just in front of it are small lamps used for flat-fielding, about which more explanation will be given later again.

Software

Here we overview the main functions of onboard AMICA software now under development, though some of them may be subject to change before launch. Since the supposed CPU for the AMICA has fairly high performance, it provides us with a capability for various image handling and reduction.

Some of them are listed as:

- Image data compression (Reversible/Irreversible).

- Automatic exposure time calculation for the set of seven AMICA filters.

- Readout smear correction operation.

- Arithmetic operations between 16 images in the local frame-memory.

- Calculation of some averages (Mean/Median/Mode values) over a specified region of CCD.
- Output of pixel-signal histogram.

- Cutout of partial images.

- Software pixel binning.

- Simple automatic aperture photometry of a stellar image in the AMICA field-of-view.

These functions and operations can be activated by the uplink commands whenever necessary.

\section{Calibrations}

Instrument calibrations are very important for acquiring scientifically meaningful results from the observed data. However, we cannot perform elaborate calibration procedure on spacecraft, otherwise possible on the ground, because of tight constraints of hardware resources and severe inflight environments. Therefore, onboard calibration methods are preferable to be as simple and robust as possible. We briefly describe photometric calibration and flat-fielding in the following.

\section{Photometric and geometric calibrations}

As already mentioned in Section 3, AMICA makes photometric observations of the target asteroid referred to the ECAS system; past asteroid missions have never adopted such an approach. For the purpose, we will follow the following procedure.

Although seven AMICA band-pass filters were fabricated, in terms of central wavelength and FWHM, by mim- 


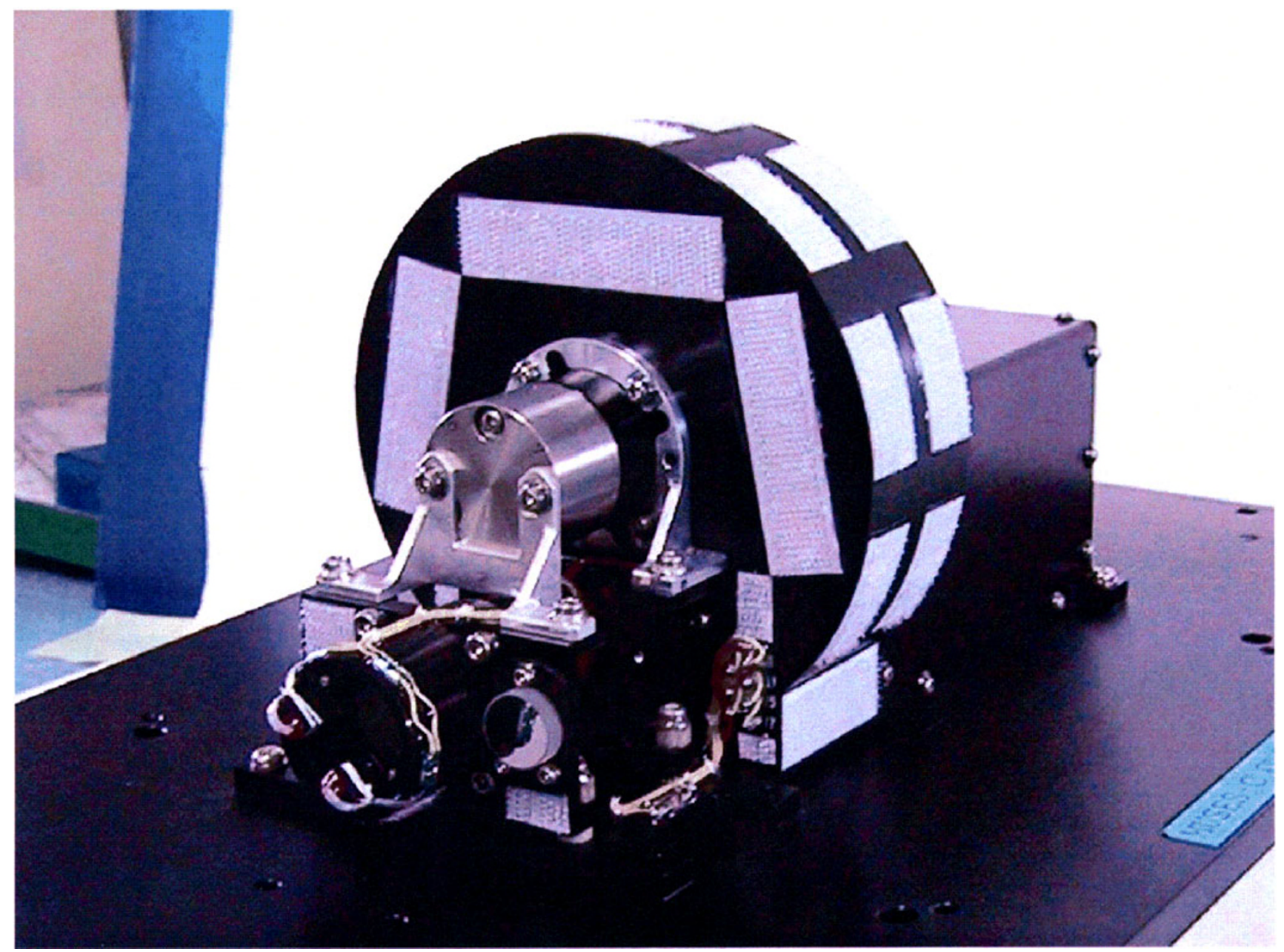

Fig. 6. Photo of the AMICA proto-model assembly. The black tube in the lower front part is the refractor telescope. As for two protrusions at the telescopic part, see text.

icking those for the original ECAS system (Tedesco et al., 1982), it was impossible to have a perfect match (compare Fig. 5 with the original ECAS band profiles). Additionally, the AMICA detector and optical system are different from the original ECAS hardware; it adopted a photomultiplier whereas we use a CCD. Hence, we need to determine magnitude conversion coefficients between the original ECAS and the AMICA system, for each band. This must be done by observing some of the ECAS standard stars. Unfortunately, however, the original ECAS standard stars are all so faint (ranging from 9th to 12th magnitudes in V-band) that the AMICA cannot observe them directly.

Therefore, as the first step, we ourselves had to produce intermediary standard stars of 4-5th magnitudes (here called the AMICA standard stars) referred to the ECAS system, by making their ground-based observations. Our AMICA standards comprise several stars ranging from G-type to $\mathrm{M}$ type in spectral classes. Once magnitudes of those stars are expressed in the ECAS system through the observations on Earth, we can use them inflight as if they were the original ECAS standards. Calculations showed that, even for the AMICA standards, low-sensitivity filters like ul- and zsbands require exposures of more than several ten seconds to obtain adequate $\mathrm{S} / \mathrm{N}$ ratio. This is a reason why a long- exposure mode is necessary for the AMICA (see Table 1). The photometric calibration procedure explained here will allow us to compare the observed surface color of 1998SF36 with the rich samples accumulated in the ECAS asteroid database.

In order to get reflectance spectra of an asteroid, the observed output from each band should be divided by the solar spectrum corresponding to the band. For the purpose, we plan to make onboard observations of a solar-analog star. A flux standard star will also be observed to perform an absolute calibration.

Calibrations for polarization measurements must be done most carefully, because expected polarization degree of the target asteroid will be small for small Sun-asteroid-spacecraft angles (or solar phase angles) and the accuracy of polarization degree measured with glass polarizers is generally said to be no better than $0.5-1 \%$ even with enough photons (e.g., Chanan and Helfand, 1990). One of the authors (T.N.) also had similar experience in comet polarimetry (Nakamura et al., 1991). In such cases, possible instrumental polarization may easily contaminate the true signal, particularly when it is weak.

Our four glass polarizers are placed with position angles of $0^{\circ}, 45^{\circ}, 90^{\circ}$, and $135^{\circ}$. Let the outputs from these po- 


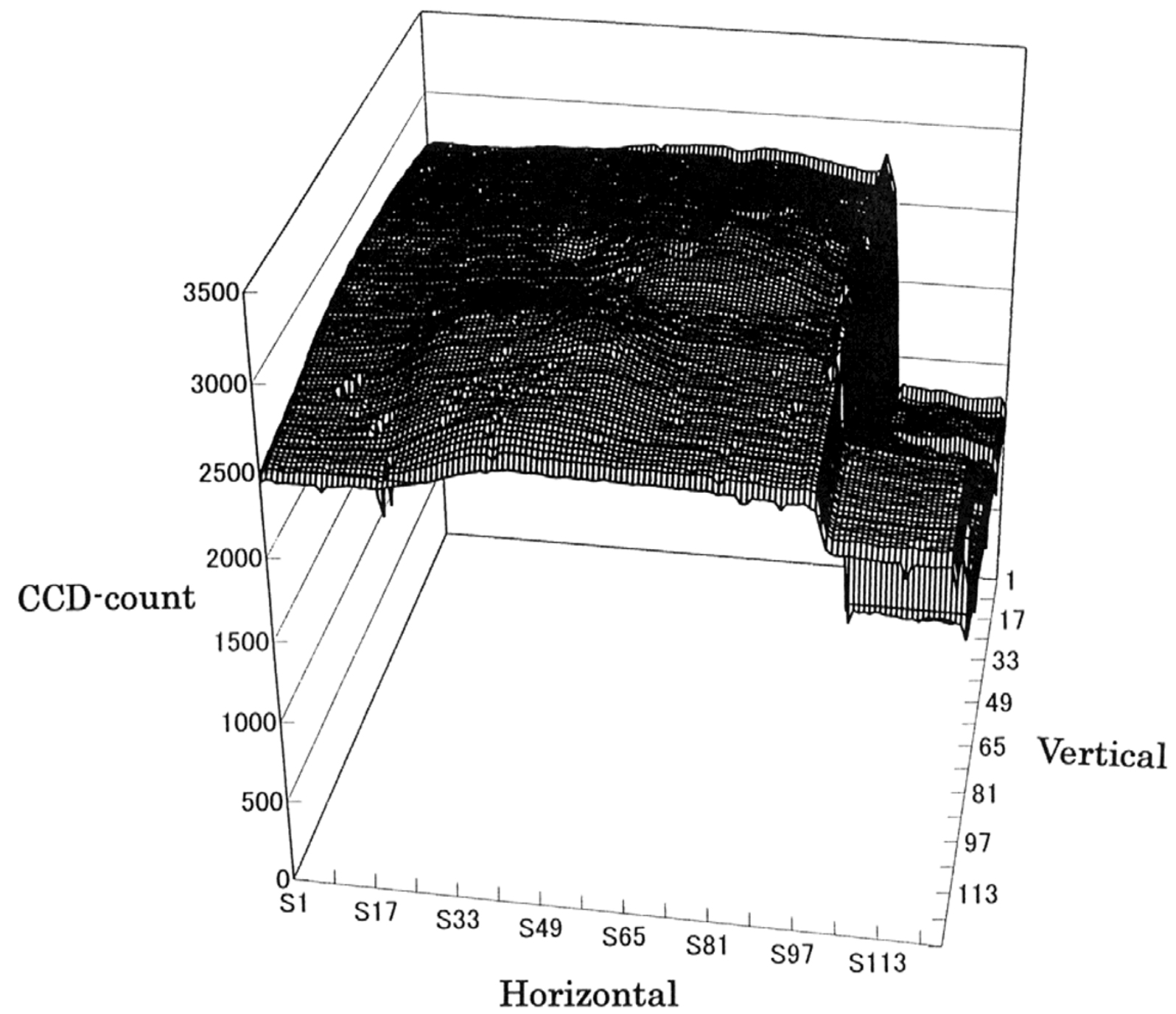

Fig. 7. An example of CCD output from the lamp-flat device. The ordinate is the digital number as a result of 12 -bit A/D conversion. The signal depression on the right-hand side is caused by four glass polarizers. The RMS variation from an ideal flat was found to be about $5 \%$ in this case.

larizers be $I_{1}, I_{2}, I_{3}$, and $I_{4}$, respectively. Then, we can calculate the polarization degree $(p)$ and polarization angle $(\phi)$ via Stokes' parameters ( $Q$ and $U$ ) through the following equations:

$$
\begin{gathered}
Q=\left(I_{1}-I_{3}\right) /\left(I_{1}+I_{3}\right), \\
U=\left(I_{2}-I_{4}\right) /\left(I_{2}+I_{4}\right), \\
p=\sqrt{Q^{2}+U^{2}},
\end{gathered}
$$

and

$$
\phi=1 / 2 \cdot \arctan (U / Q) .
$$

To remove the AMICA instrumental polarization, we observe onboard a standard star of strong polarization and determine the instrumental corrections $\Delta Q\left(=Q_{c}-Q_{o}\right)$ and $\Delta U\left(=U_{c}-U_{o}\right)$, where the subscripts $c$ and $o$ respectively stand for a catalog and observation values of the star. When these corrections $\Delta Q$ and $\Delta U$ are applied to the observed Stokes' parameters of the target asteroid, we will have true polarization of the asteroid through Eqs. (3) and (4).

Finally, in order to make a global color mapping in good accordance with topological features of the asteroid, precise geometric calibration of the CCD field-of-view is also required. This will be attained by observing two open clusters near the ecliptic, Pleiades and Hyades, for which relative astrometric positions of the member stars are known very precisely from star catalogs.

\section{Flat-fielding}

Flat-fielding is particularly important in imaging the surface of the asteroids like 1998SF36, because it will be hard for us to anticipate a distinct color variation over the surface of such small asteroids. At the initial stage of the AMICA development, we had assumed a combination of the AMICA on a steerable gimbal with a sun-illuminated white calibration board, an orthodox approach used by the Voyager spacecraft, for instance. However, subsequent strict weight constraints for the MUSES-C spacecraft as a whole made it impossible to adopt such a heavy option. Light-weighted solutions for flat-field without movable parts were really needed. After some trial and errors, we found out that highly defocused images of a tiny bare lamp(s) placed just in front of the AMICA quartz window glass work as a reasonably good flat for our purposes. Two protrusions seen at the AMICA telescope tube in Fig. 6 are the device for flat-fielding. 
An example of bird-eye view presentation for the flat-field output is shown in Fig. 7. The RMS variation from an ideal flat was about $5 \%$ in this case. We feel that adjustment of lamp location will give a little better results.

We realize that only the output from such a simple device is not sufficient per se as the flat-field we assume. Rather, we regard this lamp-flat as a device to monitor transmittance variation over the CCD field-of-view during the four-year cruise in space. Before launch, the original pattern of the lamp-flat is measured beforehand with respect to an integral sphere output, a supposed ideal flat. After launch, this laboratory-measured pattern is compared regularly with the inflight lamp-flat measurements, so that we can monitor possible local changes of optical system transmittance, probably caused by the solar flare irradiation and possible adhesion of artificial dust emitted from the launching rocket.

This lamp-flat device is also useful to measure variation of the output ratios between AMICA filters. Gammaray source exposure experiments revealed that a total dose equivalent to a four-year space flight brought about a transmission loss of the AMICA optical system by $20-30 \%$ for wavelengths shorter than $500 \mathrm{~nm}$, while there was no substantial loss at wavelengths longer than $800 \mathrm{~nm}$. Therefore, by monitoring filter transmittance relative to near-IR bands using this lamp-flat device, we will be able to measure the long-term inflight transmittance change for each filter (experiments showed that, for much longer times than an assumed inflight lamp-operation period, there was no appreciable change of the spectral transmittance).

\section{Observational Scenario and Related Sciences}

Table 2 summarizes the exploration scenario of the target asteroid along with relating sciences. For convenience, observations here are divided into four phases roughly in decreasing order of distances between the asteroid and the MUSES-C. On the left-hand column of Table 2 are given items about observing conditions such as the camera resolution and observation period, and science categories to be done.

\subsection{Cruising phase}

The cruising phase is meant here by the period from the first detection of the target asteroid which will take place approximately at spacecraft-asteroid distances of one million $\mathrm{km}$ down to the encounter with the asteroid $(\sim 20-30 \mathrm{~km})$. During the interplanetary flight before entering this phase, basic health-check, flat-field measurements of the AMICA and star cluster observations for geometric calibration will of course be performed intermittently.

Upon arrival of the MUSES-C at distances where 1998SF36 is bright enough to give high S/N ratio for photometry (a few hundred thousand kilometers), AMICA starts taking images of the asteroid every 20-30 min. for a few weeks, to obtain its continuous lightcurve. From groundbased observations at a specified site, such uninterrupted long-duration measurements of light variation of an asteroid can never been attained due to the daily change of day and night.

The free nutation (non-principal axis rotation) of an asteroid was clearly identified for the first time by the analysis of radar images of the near-Earth asteroid, Toutatis (Hudson and Ostro, 1995). According to Burns and Safronov (1973), and Harris (1994), it is shown that a dumping timescale of the free nutation of an asteroid induced by meteorite impacts is inversely proportional to the square of its size and the cube of the spin rate. Therefore, there is a much higher chance of detection of free nutation for a $\mathrm{km}$-sized asteroid like 1998SF36 (in particular if it has a long rotation period), than the large asteroids observed by the US spacecraft. Since the free nutation period is supposed to be longer than the spin period in general, lightcurve data covering an extended period of time are required for the nutation-period search using Fourier analysis, for instance. This is the reason that we are interested in the uninterrupted long-term lightcurve. Observations of disk-integrated colors of the target asteroid during the phase is also important, because such data works as an intermediary connecting in-situ measurements with groundbased observations.

Another interesting science in this phase is a search for possible satellite(s) around the target asteroid, as demonstrated by the discovery of Dactyl orbiting the asteroid Ida (Veverka et al., 1996; Belton et al., 1996). Satellite search should be attempted also in the MUSES-C mission, as there is no a priori reason to deny a satellite(s) around $\mathrm{km}$-sized small asteroids. It will be the best strategy to look for possible satellites at distances where the AMICA field-of-view is nearly equal to the apparent size of Hill's stability region of satellite motion in the restricted three-body problem (e.g., Moulton, 1914). In case of 1998SF36, with the assumed $1 \mathrm{~km}$-diameter and mean density of 3 (cgs), the search chance takes place at distances of 660-1300 km. Given the limiting magnitudes for the AMICA to be 5 mag in V-band, the detectable minimum diameter of satellites is found to be 2-3 $\mathrm{m}$ for S-type and 3-7 $\mathrm{m}$ for C-type respectively.

\subsection{Global Mapping Phase 1}

In this phase, the MUSES-C spacecraft will occupy the so-called "Home Position" (HP) for the most part. HP is characterized by the small region near the target asteroid, roughly on the line connecting the Earth with the asteroid and on the Sun side. Throughout about five months' stay of the MUSES-C in this phase, the Earth-spacecraft-Sun angle does not exceed 20-25 degrees, so that the phase angles of the asteroid observed from the MUSES-C is not large either. The spacecraft height from the asteroid surface at the HP is kept around 10-20 km (Table 2). Choice of such heights is due to the requirements that the MUSES-C should be outside the sphere of influence for the asteroid, to save fuel consumption for the heliocentric orbit keeping.

The majority of AMICA sciences will be done from the HP. They include 1) global topographic and colorimetric mappings, which are important for both physical characterization of the asteroid surface itself and selection of sampling sites, 2) measurements of the overall shape and spin state, 3) photometric and polarimetric behavior of the asteroid near opposition, and 4) validation of the asteroid nature obtained from ground-based observations. In the following paragraphs, some of them are explained more in detail.

Morphological sciences

The bulk density of an asteroid is one of the most fundamental quantities characterizing the asteroid material and 


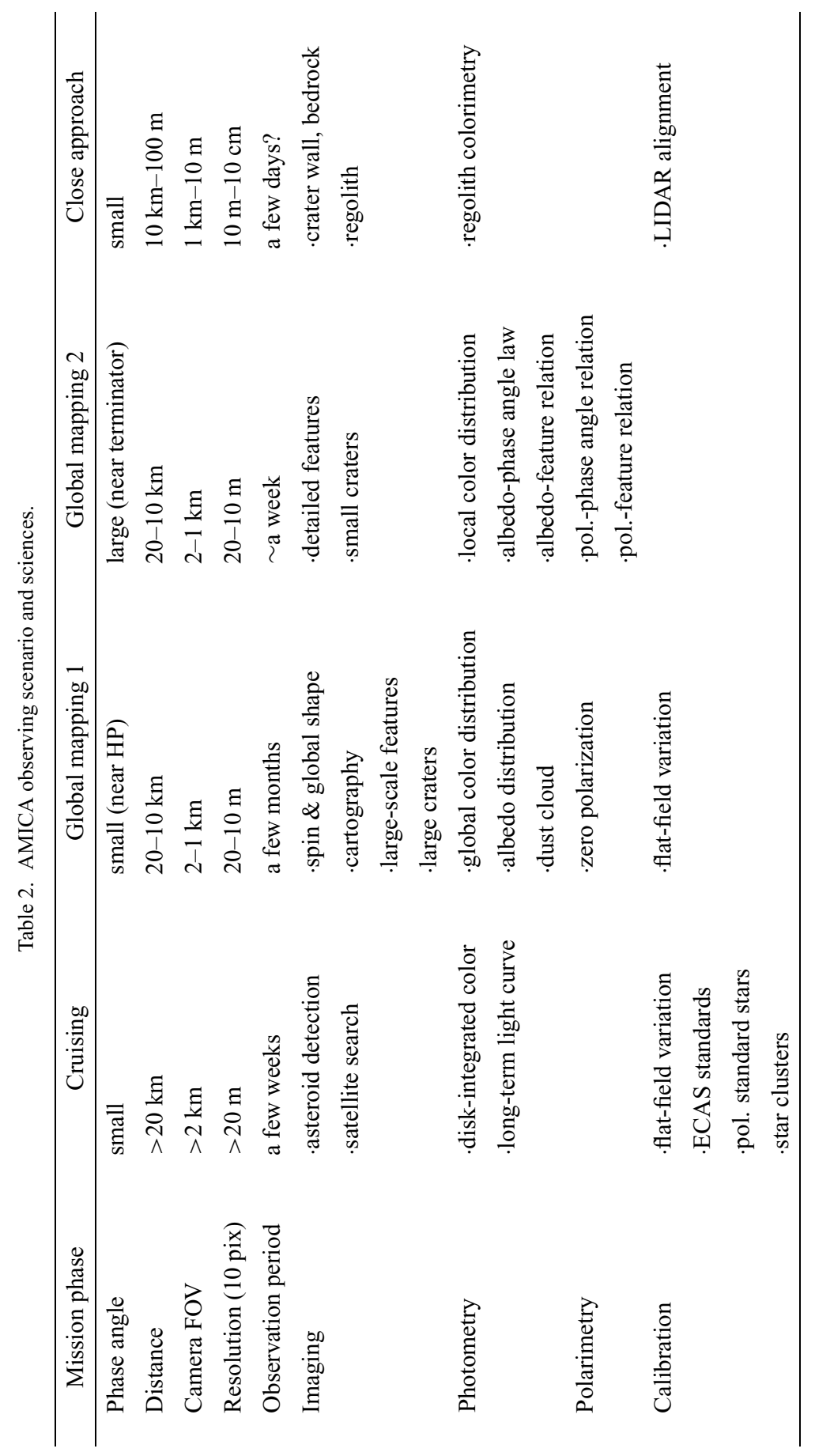



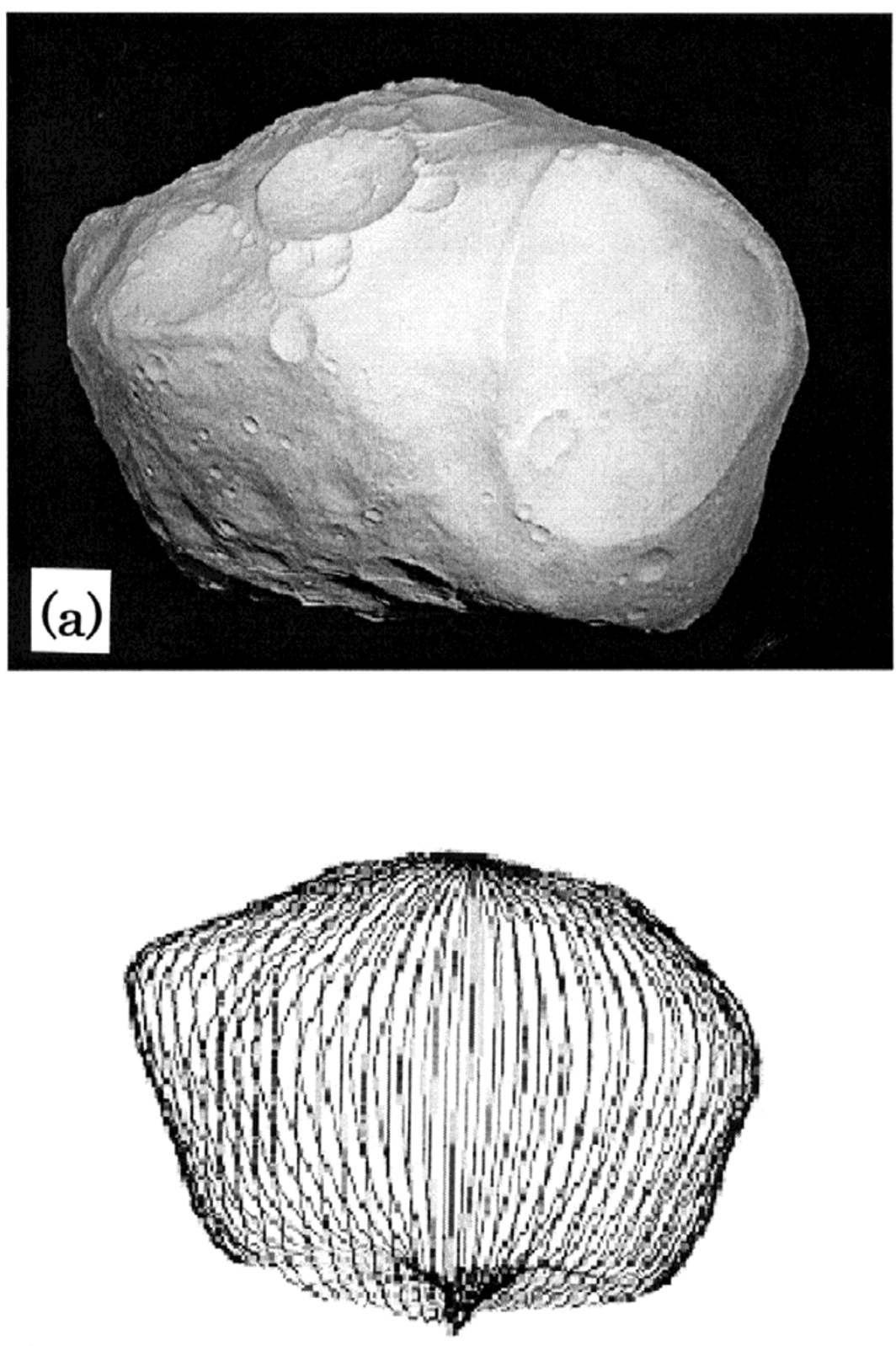

(b)

Fig. 8. (a) A stereo photo of a clay scale-model for the Martian satellite, Phobos. This model was used in our simulations for global shape measurements of an assumed asteroid. (b) A wire-framed representation reproduced from the terminator-line measurements of the Fig. 8(a) scale-model. Measurements were made by five-degree longitude interval along the equator.

internal structure. The bulk density needs measurements of mass and global shape (or volume), the latter of which should be observed with the AMICA; another means for measuring the bulk density could be LIDAR (see Mukai et al., 2001). Since the time and image number necessary to complete the global surface mapping of 1998SF36 depend complicatedly on the spacecraft location relative to the Sun and the asteroid spin state, we cannot rely on a single method of observations but will have to use a combination of the following approaches:

- Profiling of the Sun-illuminated terminator lines.

- Stereo photo mapping.
- Control-point network matching.

- Photoclinometry using shading models.

- LIDAR scanning.

Figure 8(a) is a photo of a clay scale-model of the Martian satellite Phobos that is intended for use in our simulations. As an example, we applied the first of the approaches listed above to the Phobos model. Figure 8(b) stands for a wireframed representation of the model, which was reproduced through repeatedly imaging it by five-degree longitude interval along the equator. The error in the volume estimate was found to be less than 3-5\% (Saito and Akiyama, 1996). Testing of other methods to be applied to the scale-model 


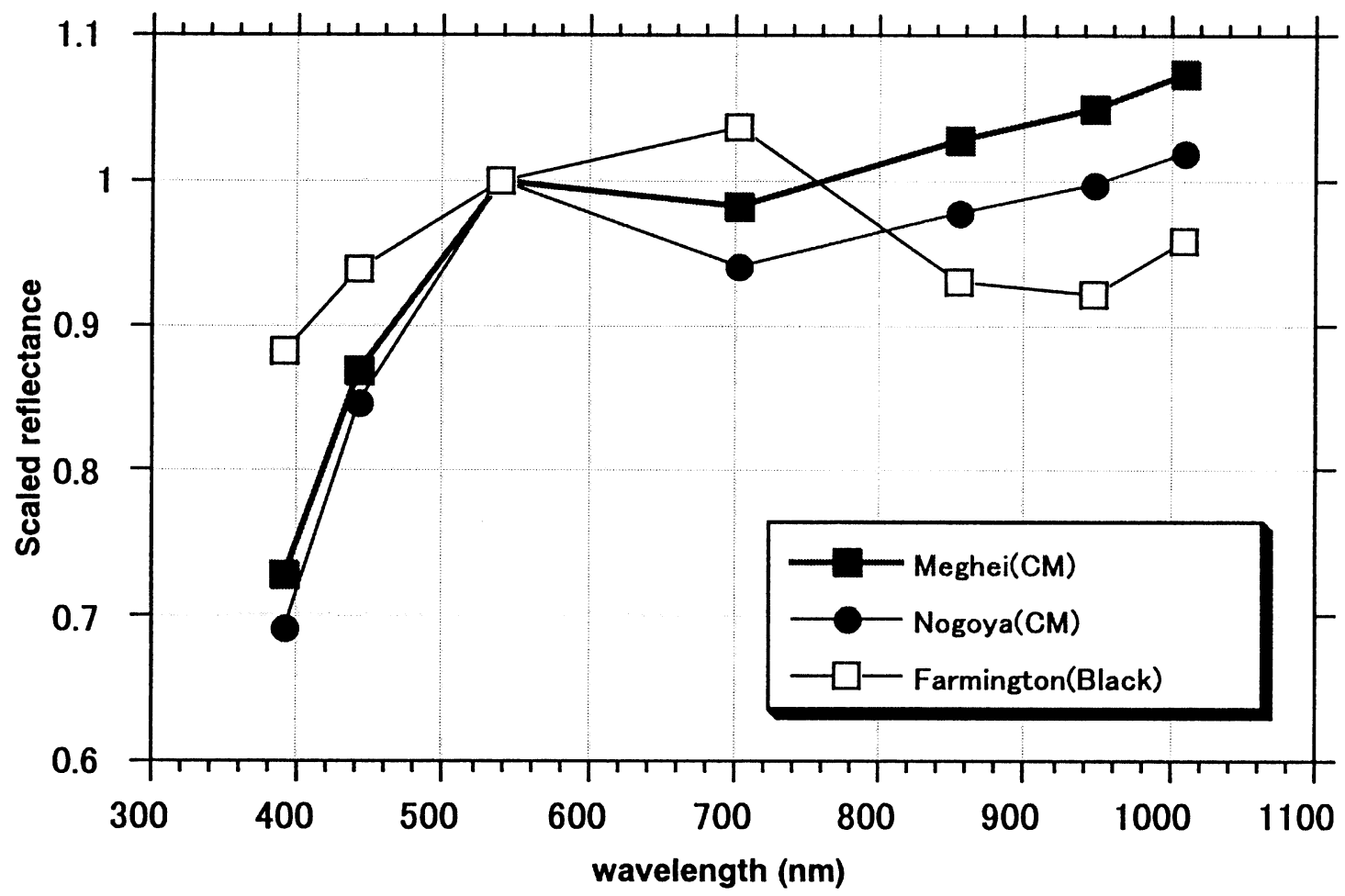

Fig. 9. Absorptions caused by CM carbonaceous chondrites and "black" ordinary chondrites as seen by the AMICA filters. The squares and circles are for the AMICA bands. The input data are based on Gaffey (1976) and also available at the PDS spectroscopy subnode (http: //www.planetary. brown. edu/planetary/pds/).

for global shape measurements is now under way.

Other important morphological studies are detailed observations of topological features such as structure of large craters, grooves and ridges if any, crater count as a function of size, sizable impact remnants on the surface, regolith layer possibly exposed at large crater walls, and so on. Helfenstein et al. (1994) showed that color distribution correlates with regional topography on the surface of the asteroid Gaspra observed by the Galileo spacecraft. In this respect, whether such a trend can be seen or not on the surface of small asteroids like 1998SF36 is an interesting issue also for the AMICA. However, those observations are not appropriate from the HP, since phase angles are not large there enough to provide high contrast imaging (except a skewed narrow area of the terminator). Hence, such sciences will mainly be done on the way to or near the Global Mapping Phase 2.

Surface color vs. geology and mineralogy

It is one of the most important themes for spacecraft to make an in-situ mapping of surface colors for a target asteroid, because they bear geological and mineralogical/petrological evidence for both the origin and the subsequent thermal/impact evolution of the asteroid. Such a map should be useful for selecting sampling locations for the MUSES-C mission as well. Color mapping does not need the high spatial resolution that requires large phase angle imaging, so that this science could be done in this Global Mapping Phase 1. After returning the captured samples to the Earth, they are subject to various scrutinies from spectroscopic measurements through isotope analysis. Comparison of the results with the color map observed by the AMICA will then enable us to do the so-called "ground-truth" study of the asteroid, as Earth observation satellites and the Apollo missions have made very successful achievements in a similar approach.

In order for the ground-truth study to work effectively for 1998SF36, one must beforehand have good knowledge of how candidate meteoritic minerals on the target asteroid look like as reflectance spectra, particularly in terms of absorption features and reddening. Unfortunately, however, almost nothing has so far been known about the physical nature of 1998SF36. Therefore, at present, we now carry out simulations of the AMICA multi-band observations by assuming a wide variety of possible meteorite analogs.

Here are some examples from our extensive simulations. Figure 9 shows the simulated spectra as measured by the AMICA filters, for CM carbonaceous chondrites and "black" (heavily shocked and darkened) ordinary chondrites. The squares and circles in the figure correspond to the AMICA bands. Visible-NIR meteorite spectra taken from the NASA-PDS spectroscopy database were used as the input data. One can see the characteristic absorption caused by phyllosilicates around $700 \mathrm{~nm}$ and a shock-affected weak absorption of mafic silicates (olivine and pyroxene) for the black chondrites (near $900 \mathrm{~nm}$ ). If there are such materials on the target asteroid, the AMICA could identify them, at least to some extent.

Another interesting issue of asteroid spectroscopy is that for metamorphism of low-albedo asteroids. There has been a controversy on how C-type and its subclass asteroids are 


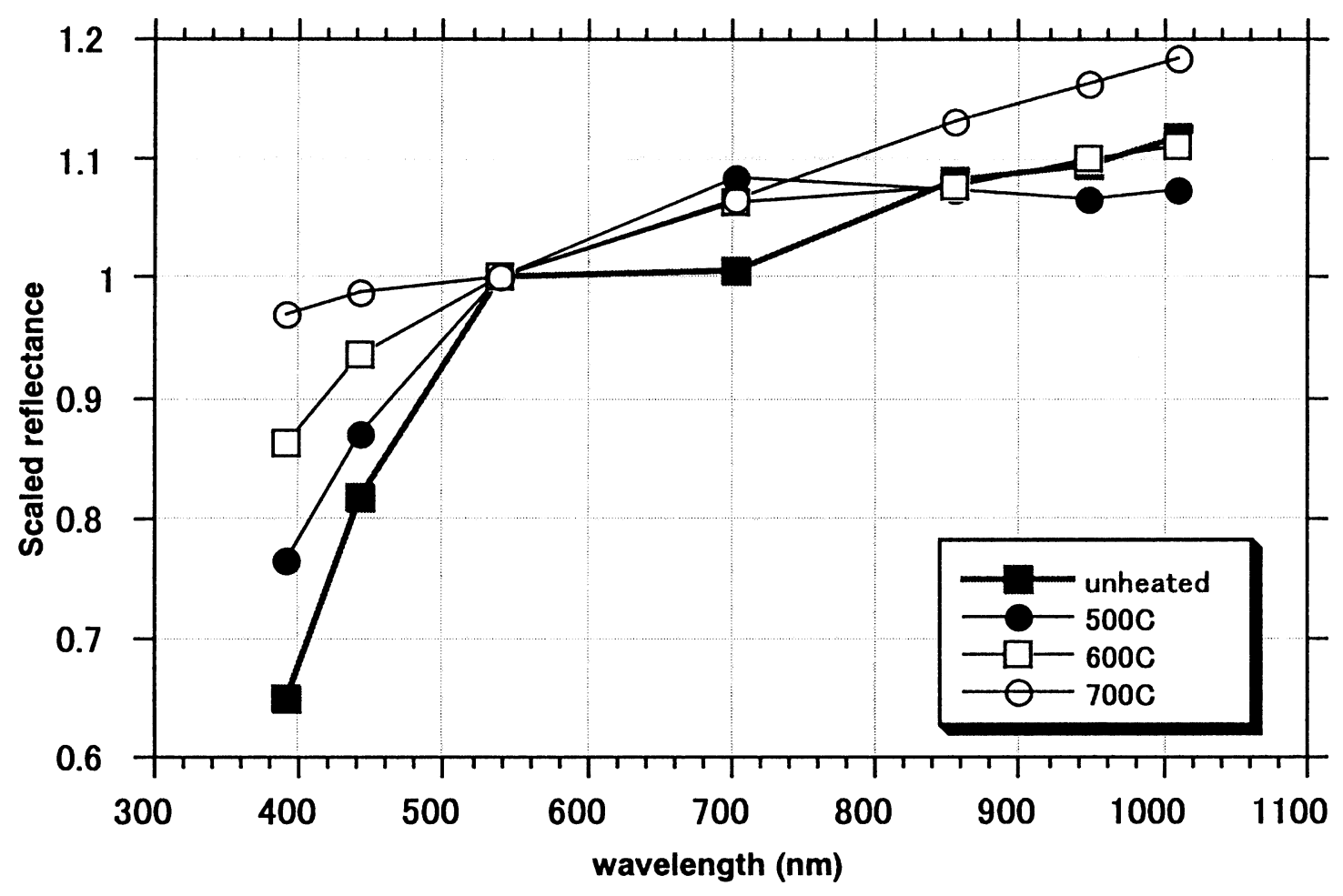

Fig. 10. AMICA-simulated spectra for Murchison CM carbonaceous chondrites heated in vacuum at various temperatures. The input data were taken from Hiroi et al. (1994). If spectral features of low-albedo asteroids reflect thermal metamorphism, AMICA ul-, b-, and v-bands may easily distinguish degree of the metamorphism.

related to the CI and CM carbonaceous chondrites, postulated spectral analogs, in terms of metamorphism. Hiroi et al. (1994) reported that spectra of Murchison CM chondrites heated in vacuum at certain temperatures have a good resemblance to those of C-, G-, B-, F-type asteroids, suggesting the evolutionary relationship between those meteorites and asteroids. On the other hand, Vilas and Sykes (1996) recently addressed a question on validity of the suggestion by Hiroi et al., by evidencing behavior of the spectral feature at $700 \mathrm{~nm}$ against heating processes, which Vilas and Sykes claim to contradict with the evolutionary scenario by Hiroi et al. (1994).

Figure 10 plots our simulated spectra at ECAS bands of Murchison CM chondrites obtained in the heating experiments by Hiroi et al. (1994). From this figure, we see that our AMICA filter system can easily detect both the $700 \mathrm{~nm}$ spectral feature pointed out by Vilas and Sykes (1996) and a general trend by Hiroi et al. (1994) that more heated CM chondrites have a gentler spectral slope toward shorter wavelengths, if any. Hence, combination of laboratory mineralogical analyses of MUSES-C returned samples with AMICA spectra may hopefully give clues to settle the opposing discussions by the above two groups.

In case of the target asteroid being of S-type, observations of the degree of so-called "space weathering" are also important; there is some discussion that S-type asteroids are masqueraded parent bodies of ordinary chondrites, whose surfaces are space-weathered by bombardments of micrometeorites so that the spectra show steep reddening toward longer wavelengths (Chapman, 1996). Yamada et al. (1999) recently conducted laboratory experiments to support the idea of space weathering. If the target asteroid reveals any appreciable reddening in its spectrum, the AMICA filter system will easily be able to detect it.

\section{Other sciences}

The surface of airless small objects is incessantly subject to hypervelocity meteoroid bombardments, probably resulting in formation of dust cloud around them. Formation of dust rings around the Martian satellites has been theoretically discussed by many investigators (e.g., see Ishimoto, 1996 and references therein). Although there are no such studies yet regarding small asteroids and the dust cloud formation mechanism can probably be different from that for the Martian satellites, it is possible that asteroids have a similar dust cloud. Considering that a greater impact meteoroid flux necessarily implies production of a higher cloud density and a higher chance of its detection, Nakamura (1998) proposed an in-situ exploration of dust cloud enhancement around mission target asteroids during their encounters with meteor showers, in which the impact flux sometimes surges up by a factor of 100-1000 compared with the steady-state flux. He calculated encounter chances of some existing spacecraft with major meteor showers.

Spacecraft observations of dust cloud produced by meteor stream impacts on an asteroid are quite important from the following two reasons. The first is that optical measurements of dust cloud density will give a clue to impact regolith formation mechanism on the low-gravity surface of small asteroids. The second is that detection of an enhanced dust cloud can ascertain the accuracy of the relevant shower 


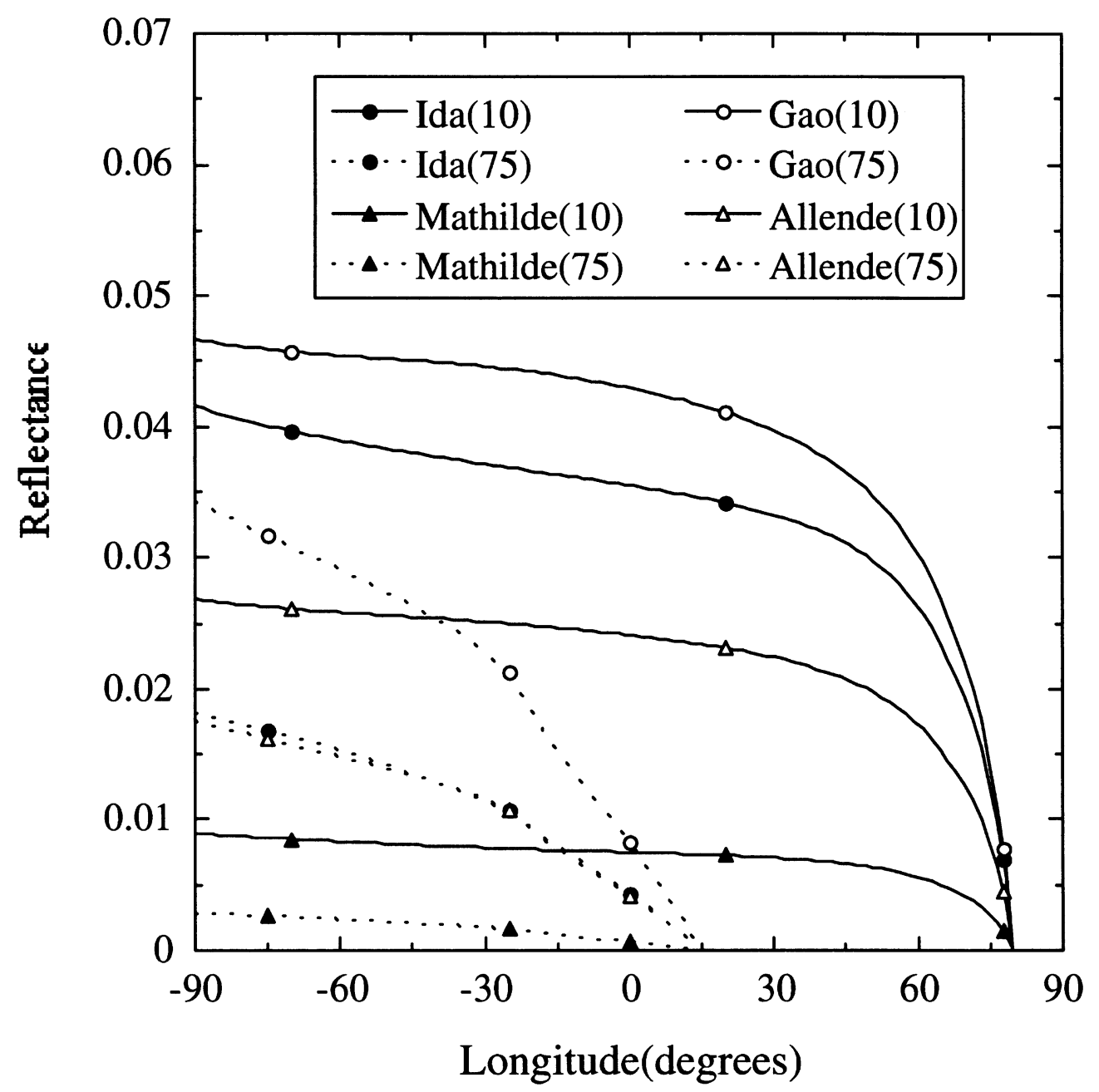

Fig. 11. Bidirectional reflectance curves for asteroids and meteorite powders as a function of longitude. The longitude is measured along the luminance equator, as defined in Hapke (1993). Solid lines are at the phase angle of $10^{\circ}$, and dashed lines at the phase angle of $75^{\circ}$ (these angles correspond to parenthesized numbers in the inset-box of the figure). Filled circles correspond to the Hapke parameters for the asteroid Ida (Helfenstein et al., 1996), filled triangles for Mathilde (Clark et al., 1999), open circles for 180-500 $\mu \mathrm{m}-\mathrm{Gao}$ (H5) powders, and open triangles for 180-500 $\mu \mathrm{m}-\mathrm{Allende}$ (CV3) powders (Kamei and Nakamura, 2000). The data for the meteorite powders are based on the laboratory measurements, except for the macroscopic roughness parameter $\bar{\theta}$, which is assumed here to be $25^{\circ}$. This is because, in the laboratory experiments, the sample surfaces were known to be more flattened than on the asteroid surface; disk-resolved data for asteroids showed the macroscopic roughness of around $25^{\circ}$, such as $29^{\circ}$ for Gaspra and $18^{\circ}$ for Ida (Helfenstein et al., 1994, 1996).

orbits and their internal meteoroid distribution, which have been determined only from very limited orbital arcs near the Earth. The AMICA will monitor the sky brightness near the edge of the target asteroid, particularly during the times when the MUSES-C encounters with meteor showers are predicted.

We have mentioned in Section 4 that transmittance of the AMICA optical system can deteriorate due to the cosmicray exposure. Hence, observations of the AMICA standard stars should preferably be made near the target asteroid. For the purpose, we selected the stars that can be observed in the Global Mapping Phase 1.

\subsection{Global Mapping Phase 2}

As shown in Table 2, this phase covers the period for the MUSES-C on the way going to and near the terminator of the target asteroid, where phase angles are large up to roughly 90 degrees. Sciences to be done with high priority in this phase are observations of detailed topography and photopolarimetry. The former will allow us to obtain crater statistics down to the AMICA-resolved sizes and a possible color vs. regional feature correlation.

\section{Photometry and Polarimetry}

Photometric property of the light scattered by an asteroid surface is sensitive to both its composition and physical structure, such as large scale (the scale larger than camera resolution) slope, roughness and porosity of the surface, and/or particle size distribution on it. In analyzing the photometric data of asteroid surfaces, Hapke's bidirectional reflectance model (Hapke, 1993) has most widely been used. From numerical simulations and laboratory experiments (Hartman and Domingue, 1998; Kamei and Nakamura, 2000), it has been shown that a wide coverage of phase angles is essential to retrieve reliable parameters relating, directly or indirectly, to the physical structure of the surface. Figure 11 shows examples of bidirectional reflectance curves for some asteroids and meteorite 


\section{Distance $=20 \mathrm{~km}$}

\section{Sub SKC longitude $=30$ degree}

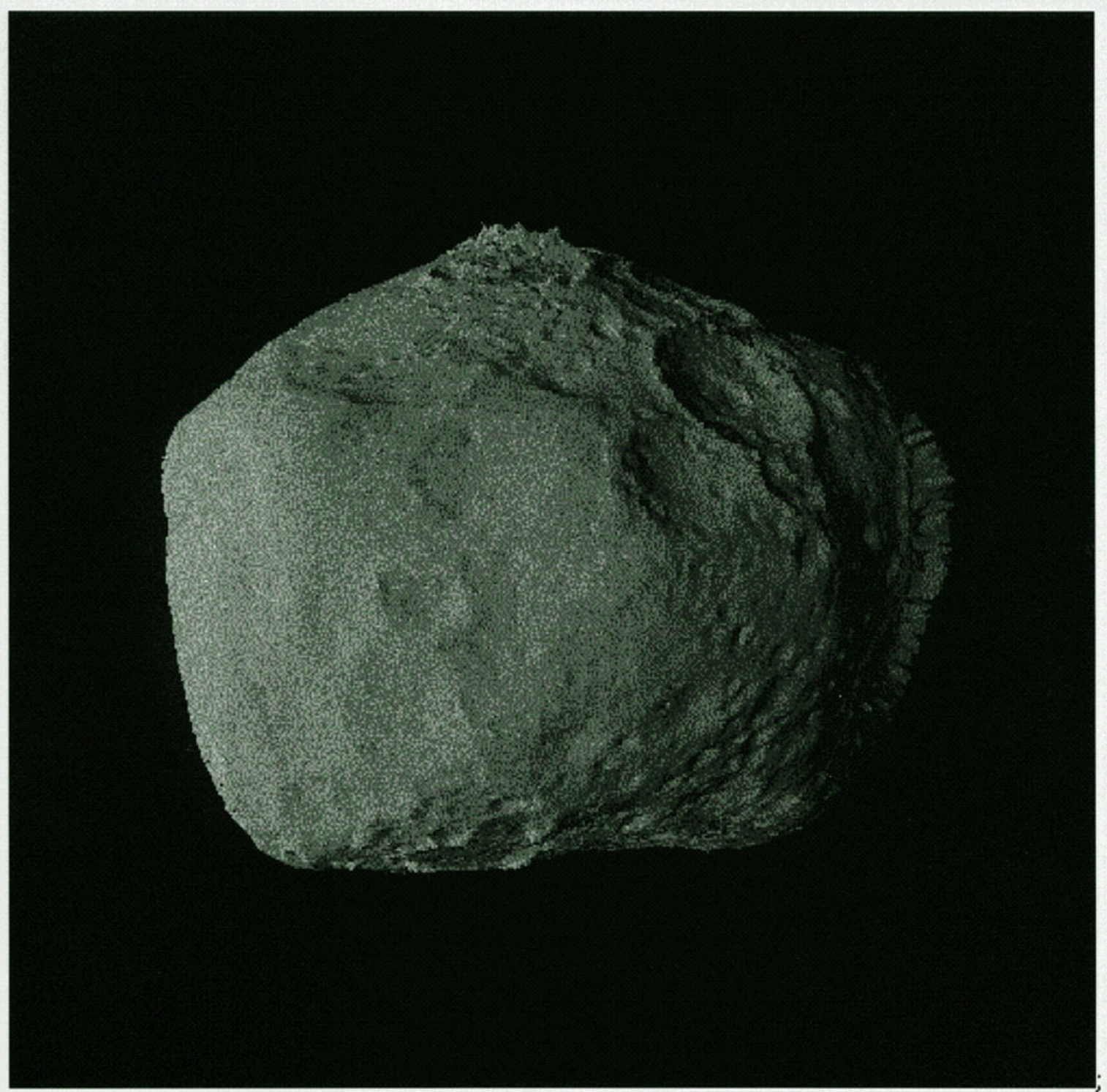

Fig. 12. An example from the AIS output. Photometric parameters for the Martian satellite Phobos are assumed.

powders. They are calculated using the Hapke's parameters determined by spacecraft observations and laboratory experiments. Two solar longitudes, $-10^{\circ}$ and $-75^{\circ}$ which correspond to phase angles of $10^{\circ}$ and $75^{\circ}$ are assumed for each case. The origin of longitude is taken here to be the sub-camera point on the observed surface.

Usefulness of those reflectance curves will be twofold. First, if a bidirectional reflectance curve is obtained in the MUSES-C mission, we may judge what asteroids/meteorites the surface of the target asteroid resembles, based on such simulation curves. Second, given the taxonomic type of the target asteroid, model reflectance curves will enable us to specify appropriate exposure times for each AMICA band over the entire disk of the asteroid.

The small scale structure, roughness, porosity, and/or particle size distribution on the asteroid surface affect not only the bidirectional reflectance but also the polarization vs. phase curve. A typical polarization curve of an asteroid is characterized by the following behavior. Linear polarization degree $(p)$ of the asteroid surface starts from zero at the phase angle $\alpha=0^{\circ}$, and is kept negative up to the inversion angle, typically $\alpha=20^{\circ}$. Then, beyond the inversion angle, the curve turns positive and increases monotonously until it reaches the maximum, $p_{\max }$, at $\alpha \sim 100^{\circ}$.

Darker asteroids generally show larger $p_{\max }$, i.e., this implies that $p_{\max }$ is approximately inversely related to albedo. 
Dollfus (1998) revealed that the relation between $p_{\max }$ and albedo is dependent upon the particle size of the surface. Hence we expect to have a clue on the particle size using the observed $p_{\max }$ and albedo. The observational coverage of phase angles in the Global Mapping Phase $2\left(0^{\circ}<\alpha<\sim\right.$ $\left.90^{\circ}-100^{\circ}\right)$, however, might not be adequate for determining a reliable $p_{\max }$, since observable time is severely limited particularly at $\alpha>50^{\circ}-60^{\circ}$ due to electric power constraints relating to the attitude of solar-cell array panels. Therefore, we will also measure the slope of the polarization-phase curve at phase angles larger than the inversion angle, in an attempt to give a constraint on the particle size of the surface by use of the relation between the slope and albedo (Kamei, 2000). By combining all the photopolarimetric data with the color and topography maps for the target asteroid, we will eventually clarify possible relationships between color, regional topographic feature, small scale surface structure, and so on.

\subsection{Close approach phase}

The phase is meant by the period when the MUSES-C starts descent from the HP down near the asteroid surface for sampling. Although, around the end of this phase, we plan to make detailed imaging of small craters, minute features, bedrock and regolith structure, etc. using the closeup lens, high contrast images may not be available because of small phase angles. In addition, this close approach phase will not be so appropriate for the AMICA multi-band observations, since the spacecraft is busy looking for accessible sampling sites and preparing for the sample catch. At the heights of a few hundred meters, we will attempt to take images of LIDAR-illuminated spots on the asteroid surface with zS-filter. This is for alignment confirmation of LIDAR and AMICA. If LIDAR spots with a good $\mathrm{S} / \mathrm{N}$ ratio are observed, they might be analyzed to know scattering properties of the surface regolith at the LIDAR wavelength.

\subsection{Sample analysis and importance of microscopic re- golith imaging}

It is certain that the ground-truth study of the target asteroid as a result of the sample catch will open up an unprecedented viewpoint on the origin and evolution of asteroids. Analysis of the returned samples in laboratories can surely provide detailed information on the nature of the materials which the asteroid is made of. On the other hand, the captured samples are unlikely to retain the state of how regolith particles are packed on the surface of the target asteroid whose surface gravity is extremely small. This property of regolith stacking, however, plays an important role as a mediator connecting laboratory photometry/spectroscopy with macroscopic optical properties obtained from the AMICA observations. Currently, a domestic mobile microlander with a near-sighted panoramic camera is now under consideration for a payload of the MUSES-C spacecraft. If this plan is realized, microscopic images that the microlander provides will obviously be of great value.

\subsection{Asteroid Imaging Simulator}

Due to visibility limitations of MUSES-C spacecraft from the domestic Deep Space Network, only five to six hours per day will be available for downlink transmission of all the science data. Therefore, an elaborate planning of AMICA operations is crucial to maximize the scientific return, taking into account such as exposure time setting and spacecraft attitude relative to the target asteroid. For the purpose, a software program here called Asteroid Imaging Simulator (AIS) is developed by the Kobe Univ. people of the AMICA team.

The software can output AMICA-simulated images by specifying the following information regarding the camera and the target asteroid:

- Shape model (size, axial ratio, craters, and surface features).

- Spin-state model (single-axis spin, tumbling motion).

- Photometric model (Hapke's parameters, surface roughness).

- Camera model (CCD noise, filter band, etc.).

Figure 12 shows an example from the AIS output. The size of the asteroid is taken to be about $1 \mathrm{~km}$ in diameter. The asteroid is assumed to be illuminated at the surface of $30^{\circ}$ in longitude from the subspacecraft point. Through quantitative analysis of those simulated images, we expect to establish a better in-situ observation planning and operations.

Acknowledgments. Development of the AMICA is a joint program by our science team (AMICA team) and the ISAS team which is responsible to spacecraft navigation and attitude control. We thank Profs. T. Hashimoto and T. Kubota of the ISAS team for the collaborative work. Our thanks are also due to the NEC Corporation engineers, K. Noguchi, Y. Kiso, M. Takano, H. Hihara, H. Hosoda, M. Uo, M. Ogasawara, and H. Atake of the Showa Optronics Co., whose efforts were essential in design, production, and testing of the AMICA. Discussions with Drs. A. Fujiwara, M. Abe of the ISAS, and T. Mukai of Kobe Univ. were very valuable for refining the AMICA design and sciences. Finally, we appreciate referee comments by Dr. T. Mukai and an anonymous referee, which were useful for improving the original manuscript.

\section{References}

Belton, M. J., J. Veverka, P. Thomas, P. Helfenstein, D. Simonelli, C. Chapman, M. E. Davies, R. Greeley, R. Greenberg, J. Head, S. Murchie, K. Klaasen, T. V. Johnson, A. McEwen, D. Morrison, G. Neukum, F. Fanale, C. Anger, M. Carr, and C. Pilcher, Galileo encounter with 951 Gaspra: First pictures of an asteroid, Science, 257, 1647-1652, 1992.

Belton, M. J., B. E. A. Mueller, L. A. D’Amario, D. V. Byrnes, K. P. Klaasen, S. Synnott, H. Breneman, T. V. Johnson, P. C. Thomas, J. Veverka, A. P. Harch, M. E. Davies, W. J. Merline, C. R. Chapman, D. Davis, T. Denk, G. Neukum, J.-M. Petit, R. Greenberg, A. Stoors, and B. Zellner, The discovery and orbit of 1993 (243)1 Dactyl, Icarus, 120, 185-199, 1996.

Burns, J. A. and V. S. Safronov, Asteroid nutation angles, Mon. Not. R. Astron. Soc., 165, 403, 1973.

Chanan, C. A. and D. J. Helfand, Optical polarization of the Crab-like supernova remnant 0540-693 in the large Magellanic cloud, Astrophys. J., 352, 167-171, 1990.

Chapman, C. R., S-type asteroids, ordinary chondrites, and space weathering: The evidence from Galileo's fly-bys of Gaspra and Ida, Meteorit. Planet. Sci., 31, 699-725, 1996.

Clark, B. E., J. Veverka, P. Helfenstein, P. C. Thomas, J. F. Bell, III, A Harch, M. S. Robinson, S. L. Murchie, L. A. McFadden, and C. R. Chapman, Near photometry of asteroid 253 Mathilde, Icarus, 140, 53$65,1999$.

Dollfus, A., Lunar surface imaging polarimetry: I. Roughness and grain size, Icarus, 136, 69-103, 1998.

Gaffey, M. J., Spectral reflectance characteristics of the meteorite classes, J. Geophys. Res., 81, 905-919, 1976.

Hapke, B., Theory of Reflectance and Emittance Spectroscopy, Cambridge 
Univ. Press, Cambridge, UK, 1993.

Harris, A. W., Tumbling asteroids, Icarus, 107, 209-211, 1994.

Hartman, B. and D. Domingue, Scattering of light by individual particles and the implications for models of planetary surfaces, Icarus, 131, 421448, 1998 .

Helfenstein, P., J. Veverka, P. C. Thomas, D. P. Simonelli, P. Lee, K. Klaasen, T. V. Johnson, H. Breneman, J. Head, S. Murchie, F. Fanale, M. Robinson, B. E. Clark, J. Granahan, H. Garbeil, A. S. McEwen, R. Kirk, M. Davies, G. Neukum, S. Mottola, R. Wagner, M. Belton, C. Chapman, and C. Pilcher, Galileo photometry of asteroid 951 Gaspra, Icarus, 107, 37-60, 1994.

Helfenstein, P., J. Veveraka, P. C. Thomas, D. P. Simonelli, K. Klaasen, T. V. Johnson, F. Fanale, J. Granahan, A. S. McEwen, M. Belton, and C. Chapman, Galileo photometry of asteroid 243 Ida, Icarus, 120, 48-65, 1996.

Hiroi, T., C. M. Pieters, M. E. Zolensky, and M. E. Lipschutz, Possible thermal metamorphism on the $\mathrm{C}, \mathrm{G}, \mathrm{B}$, and $\mathrm{F}$ asteroids detected from their reflectance spectra in comparison with carbonaceous chondrites, Proc. NIPR Symp. Antarct. Meteorites, 7, 230-243, 1994.

Hudson, R. S. and S. J. Ostro, Shape and non-principal axis spin state of asteroid 4179 Toutatis, Science, 270, 84-86, 1995.

Ishimoto, H., Formation of Phobos and Deimos dust rings, Icarus, 122, $153-165,1996$.

Kamei, A., Laboratory study of laser-scattered light by rough surfaces, Ph.D. Thesis, Kobe University, 2000.

Kamei, A. and A. M. Nakamura, Laboratory study of bidirectional reflectance of powdered surfaces: On the asymmetry parameter of asteroid photometric data, Icarus, 2000 (submitted).

Kawaguchi, J. and K. Uesugi, Technology development status of the MUSES-C sample and return project, IAF-99-IAA.11.2.02, 50th International Astronautical Congress, pp. 1-9, Oct. 4-8, 1999, Amsterdam, the Netherlands, 1999.

Kopal, Z., The Moon in the Post-Apollo Era, 223 pp., D. Reidel Publ. Co., 1974.

Moulton, F. R., An Introduction to Celestial Mechanics, Chap. 8, MacMillan Co., 1914.

Mukai, T., H. Araki, T. Mizuno, N. Hatanaka, A. M. Nakamura, A. Kamei, H. Nakayama, and A. Cheng, Detection of mass, shape, surface roughness of target asteroid of MUSES-C by LIDAR, Adv. Space Res., 2001 (in press).

Nakamura, T., Dust cloud enhancement around small celestial bodies due to meteor stream impacts: Application to asteroid mission, in Dynamics of Comets and Asteroids and Their Role in Earth History, edited by S. Yabushita and J. Henrard, pp. 255-265, Kluwer Acad. Pub., Dordrecht, 1998.

Nakamura, T., S. Okamura, T. Aoki, and G. Sasaki, Polarization map and dust jet of comet Austin, the 24th Lunar and Planet. Sympo., pp. 141147, edited by H. Mizutani, the Institute of Space and Astronautical Science, Sagamihara, 1991.

Saito, J. and H. Akiyama, A model of Phobos and its application to simulation study for asteroid imaging, Lunar and Planet. Sci. XXVII, 11231124 (abstract), 1996.

Tedesco, E. F., D. J. Tholen, B. Zellner, The eight-color asteroid survey: Standard stars, Astron. J., 87, 1587-1592, 1982.

Tholen, D. J., Asteroid taxonomy from cluster analysis of photometry, Ph.D. thesis, Univ. of Arizona, 1984.

Veverka, J., P. C. Thomas, P. Helfenstein, P. Lee, A. Harch, S. Calvo, C. Chapman, M. J. S. Belton, K. Klaasen, T. V. Johnson, and M. Davies, Dactyl: Galileo observations of Ida's satellite, Icarus, 120, 200-211, 1996.

Veverka, J., M. Robinson, P. Thomas, S. Murchie, J. F. Bell, III, N. Izenberg, C. Chapman, A. Harch, M. Bell, B. Carcich, A. Cheng, B. Clark, D. Domingue, D. Dunham, R. Farquhar, M. J. Gaffey, E. Hawkins, J. Joseph, R. Kirk, H. Li, P. Lucey, M. Malin, P. Martin, L. McFadden, W. J. Merline, J. K. Miller, W. M. Owen, Jr., L. Peterson, J. Warren, D. Wellnitz, B. G. Williams, and D. K. Yoemans, NEAR at Eros: Imaging and spectral results, Science, 289, 2088-2097, 2000.

Vilas, F. and M. V. Sykes, Are low-albedo asteroids thermally metamorphosed?, Icarus, 124, 483-489, 1996.

Yamada, M., S. Sasaki, H. Nagahara, A. Fujiwara, S. Hasegawa, H. Yano, T. Hiroi, H. Ohashi, and H. Otake, Simulation of space weathering of planet-forming materials: Nanosecond pulse laser irradiation and proton implantation on olivine and pyroxene samples, Earth Planets Space, 51(11), 1255-1265, 1999.

Zellner, B., D. J. Tholen, and E. F. Tedesco, The eight-color asteroid survey: Results for 589 minor planets, Icarus, 61, 355-416, 1985.

T. Nakamura (e-mail: tsuko@cc.nao.ac.jp), A. M. Nakamura, J. Saito, S. Sasaki, R. Nakamura, H. Demura, H. Akiyama, D. Tholen, and AMICA team 